\title{
ORDINACIONS DELS BARQUERS DE LA CIUTAT DE BARCELONA DURANT EL SEGLE XV
}

\author{
Margarida Tintó
}

La vida de la ciutat de la Barcelona medieval girava en gran part entorn del mar, concretament de la ribera del sector de llevant. Eren molts els interessos comercials que s'hi movien; per això a Ciutat s'estava a l'expectativa de les anades $i$ vingudes de tota mena de naus.

Ho facilitava un accés directe: el portal major de l'antiga muralla romana donava a la plaça del Blat (després de l'Àngel) i d'allí sortia el camí de mar, que conduïa als sorrals on arribaven les mercaderies transportades per les naus. Així sorgí, a redós de l'església de Santa Maria del Mar, el barri de la Ribera, que aviat es convertí en un dels més actius i habitats de la ciutat. Allí convivien nobles, mercaders, industrials i menestrals, canvistes i corredors d'orella, sense oblidar-nos d'aquells qui directament vivien del treball que proporcionava el tràfic marítim: els mestres d'aixa i els calafats que construỉen o reparaven les embarcacions; els bastaixos o macips de Ribera que portaven les mercaderies a Ciutat; i, finalment, els barquers. A aquests darrers dediquem el nostre estudi.

Mentre no hi hagué a la ciutat ni moll ni port construîts, les diverses embarcacions fondejaven a mar obert o bé a la tasca (bancal de sorra), sense aixopluc, exposades als temporals - freqüents aleshores, segons diuen els documents de l'època-, i a robatoris; fets que comportaven de retop pèrdues considerables. Calia, per tant, que es fessin amb rapidesa els treballs de càrrega i descàrrega, com també la reparació de les naus avariades per poder fer-se a la mar com més aviat millor.

Per traslladar les mercaderies des de la platja fins a les embarcacions, i viceversa, hi havia un servei de barques o gòndoles dirigit pels barquers o gondolers, que eren ajudats pels missatgers i esclaus. Els barquers eren, realment, els qui tenien per art, ofici o labor carregar o descarregar mercaderies, robes e altres havers de les naus, galees, barques e altres fustes maritimes. Tenien una reglamentació laboral molt estricta - que ja es reflecteix en el Llibre del Consolat de Mar-, com també ho era la dels 
bastaixos i la de tot el mecanisme mercantil marítim a l'Edat Mitjana, que comportava grans despeses, $\mathrm{i}$, alhora, ingressos considerables.

Es aquest un tema mol transcendent i de gran amplitud que ha fet vessar molta tinta, especialment després de l'obra de Capmany'. Per aquest motiu pensem que pot ésser-li útil qualsevol aportacio, ni que sigui petita, com si es tractés de dipositar un gra de sorra en aquesta platja de la ciutat medieval de Barcelona, tan plena d'història. Són dades tretes dels llibres gremials, resultat de les investigacions iniciades els anys que el Dr. Frederic Udina dirigi el Museu d'Història de Barcelona i m'encarregà de la sala monogràfica dedicada a «Confraries i Gremis». Sigui, doncs, aquest treball un testimoni d'homenatge i d'agraïment.

Ens ha porta al present treball la lectura d'un llibret que, amb el títol Ordinacions dels barquers de la ciutat de Barcelona 1380-1495 i procedent de la biblioteca d'Ernest Moliné i Brasès, es conserva a l'Institut Municipal d'Història de Barcelona ${ }^{2}$. Es tracta d'un petit volum manuscrit, enquadernat amb cartro, de 54 folis numerats amb xifres romanes, més un plec afegit de 6 folis amb numeració aràbiga. S'inicia, com és usual en els llibres gremials, amb una còpia del privilegi fundacional de la confraria dels barquers, de 1380; a continuació hi ha un privilegi de la reina Maria, de 1422 , i les ordinacions de 1454 i 1474 , amb una relació de les taxes previstes per al carregament i descarregament de les diverses mercaderies, robes $\mathrm{i}$ altres havers; inclou després una nota aclaridora datada el 1437, per acabar amb unes ordinacions de 1480 i 1495 sobre els barquers «vells».

Les primeres notícies documentals sobre la confraria dels barquers de la ciutat de Barcelona les trobem en un pergamí de l'Arxiu de la Corona d'Aragó, datat el 24 de juny de $1380^{3}$. Es tracta del privilegi que signa el rei Pere el Cerimoniós amb l'aprovació dels capítols fundacionals de la confraria, posada sota el patronatge de sant Pere. En honor d'aquest sant es construirà una capella a l'antic convent de Sant Agustí, avui desaparegut, al barri de la Ribera, que servirà de lloc de reunió per a l'elecció dels prohoms, $i$ on tindran els vasos sepulcrals, en els quals serà esculpit el seu senyal, una barca.

Els capitols continuen la línia d'aquells gremis constituits en dates semblants en un sentit benèfic, social i religiós: ajuda al necessitat; vetlla de malalts i de difunts; assistència a la missa el dia de la festa del sant patró, sant Pere, amb l'obligació d'assistir al dinar, que serà al mateix convent, previ el pagament de dos sous (el dinar pot ésser més car, però aleshores ho

'2 A. CAPMany r DE Montrabau, Memorias histónicas sobre la marina, comercio y artes de la antigua ciudad de Barcelona, Madrid 1779-92. Reed. Barcelona, 1961-62, 4 vol.

2 IMH, Barquers, 34-3.

3 ACA, Reg. 935, fol. 240. Vegeu P. Bofarull r Mascakó, Gremios y cofradias de la antigua Corona de Aragón, en Colección de documentos inéditos del Archivo General de la Corona de Aragón, vol. 40, Barcelona 1876, p. 197-205. 
abonarà la confraria o gremi). La quota serà d'un diner cada setmana, que els barquers pagaran personalment els dissabtes, com també els seus missatgers i els seus esclaus. El dia de sant Pere s'elegiran els quatre prohoms que regiran el gremi durant l'any.

A partir d'aquí, successius privilegis $\mathrm{i}$ ordinacions estructuren la part laboral dels barquers, que adquireix aviat unes connotacions ben diferents de les de la majoria de les corporacions artesanals de l'Edat Mitjana. D'una banda, la diferenciació clara entre confraria i ofici. Era facil entrar a la confraria, però per exercir l'ofici calia demostrar-ne la idoneïtat. Així ho expliquen les ordinacions de 1495: segonament, que volent algu entrar en la confraria per confrare e apres en lo art e offici de barquejar, com sien coses distinctes e separades, la dita confraria e lo dit art e offici, que los prohomens hagen ab cert nombre de homens per ells applicadors, qui sien del dit art e offici, mirar e examinar de la ydoneitat de aquell tal qui en dit art e offici apres de esser fet confrare entrar volra...

D'altra banda, les ordinacions de 1474 corroboren l'existència, des de vint anys enrere, de dues companyies regides per dos prohoms cadascuna que feien el jurament davant els cònsols de la llotge de la mar. Evidentment, aquestes companyies les identifiquem amb el gremi dels barquers «vells» o de sant Pere, del qual hem parlat al començament; i el dels barquers novells, fundat el gener de 1459 per Joan $\mathrm{II}^{4}$.

Aquest era un altre gremi, que tenia per patrona la Verge de la Consolací i que celebrava la festa el 12 de setembre i l'elecció dels prohoms el dia de san Bernabè (11 de juny), si bé sembla que el costum canvià l'elecció al dia de santa Tecla. Subratllem que mentre la major part de gremis continuen emprant la denominació de confraria fins al segle XVIII, els barquers, en parlar de l'exercici de l'ofici, recorren al terme companyia.

Ja hem dit abans que era molt important que la feina es fes amb rapidesa. Per tant, en moments determinats calia disposar d'ajuda, que solien prestar els esclaus. Aquest fet era motiu de polèmica ja d'antic. Les ordinacions de 1340 - abans de la constitució del gremi- prohibien que cada barquer tingués més de dos esclaus, prohibició que hom anul-là deu anys després ${ }^{5}$. En 1395 és disposat que en moments de molta feina es puguin utilitzar obrers de fora del gremi, i concretant, és a partir del 1474. que és possible tenir tants esclaus o captius com es vulgui, sempre, però, posant-ho en coneixement dels prohoms de la respectiva companyia. A la ribera hi haurà sempre un prohom de cada companyia, o bé tots quatre, responsables de les operacions.

4 ACA, Reg. 3441, fol, 127. Vegeu F. Bofarull i Sans, Gremios y cofradías de la antigua Corona de Aragón, en Colección de documentos..., cit. vol. 41, Barcelona, 1910.

5 CAPMANY, op, cit., II, 239. 
Els esclaus es comprometran a acabar l'obra iniciada, i, mai que ningú cometés furt o frau en les mercaderies, caldrà que les restitueixi o en faci efectiu el valor. Quan existejxi el dubte de qui és l'autor del delicte, serà la companyia qui compensarà les coses furtades o el frau comès.

La forma de repartir la feina entre les dues companyies serà la següent: els barquers que arribin primer a l'embarcació que cal descarregar començaran per la part que vulguin, els altres per la part restant. Ara bé: si una companyia ha finalitzat la seva comesa caldrà que ajudi l'altra. Les mercaderies que es descarreguin seran deixades lluny de l'aigua del mar, cinc passes en exut, perquè no es mullin; però el forment, el vi, la sal, la llenya i altres gèneres seran traslladats a muntanya o a les places, segons el costum.

Quant a l'ingrés, tota companyia acceptarà qui és ciutadà de Barcelona, mariner o barquer o els seus fills, juntament amb captius o sense.

No entrem, però, en el comentari de totes les ordinacions que ens fan conèixer la reglamentació laboral dels barquers, com tampoc en l'aspecte social, altrament ja coneguts ${ }^{6}$, i centrem la nostra aportacio en la relació de les taxes que els mercaders havien de pagar per la feina de carregar de la platja a la nau les diverses mercaderies o, quan era el cas, descarregar-les. Si la taxa no era feta efectiva, els barquers podien retenir penyora pel valor corresponent.

La llista que transcrivim a l'apèndix es troba en l'esmentat Llibre a continuació de les ordinacions de 1474. Ara bé: la lletra és diferent, i no porta data concreta; només, al final, hi ha una nota aclaridora, datada el 1437, sobre la classificació d'embarcació coberta o descoberta i la forma de desembarcar determinats productes.

Diverses citacions documentals fan referència al recaptament dels salaris a percebre per la càrrega o descàrrega de cadascuna de les mercaderies, robes $\mathrm{i}$ altres havers, $\mathrm{i}$ a la seva distribució. Així, Joan I concedeix al gremi de barquers de Barcelona, el 20 de juny de 1395, llicència per poder elegir un recaptador i distribuildor dels salaris? ${ }^{7}$ La reina Maria, en el privilegi de $1422^{8}$, dóna certes normes per a la recaptació dels salaris i perquè sigui el cullidor el qui reparteixi els ingressos.

Les ordinacions de 1454 estableixen que, de tota fusta en què el sou de les descàrregues estigui per damunt dels 25 sous, correspondran a cadascun

${ }^{6}$ BonAssIE, La organización del trabajo en Barcelona a finnes del siglo XV. Universidad de Barcelona, Instituto de Historia Medieval, CSIC, Barcelona 1975, p. 130-132. Estudia l'aspecte de socors mutus dels barquers a través de les ordinacions de 1480 i 1495 ja publicades per Campany, C. CARRÉRE, Barcelona 1380-1462, un centre econòmic en època de crisi, Barcelona 1977, vol. I, p. 84-89, Comenta amb detall les ordinacions de 1454, en les quals s'articula l'estatus laboral.

7 CAPMANY, op. cit. II, 380.

${ }^{8}$ A. DURAN I S ANPERE, Barcelona i la seva història, II. La societat il'organilzació del treball, Barcelona 1973, p. 479-481. 
dels quatre prohoms 12 diners, a més de la quantitat normalment establerta. Per a una més recta distribució dels guanys, tant dels barquers del gremi de sant Pere, com dels barquers novells, estableixen també que no es puguin unir o agabellar guanys, sinó que per a cadascuna de les companyies hi haurà un escrivà cullidor e reebedor dels guanys, a fi de poder compartir les quantitats rebudes entre tots aquells qui han participat en els treballs. Participaven igualment en la distribució aquells qui ja no podien treballar per l'edat $i$ els malalts. No es limitava el temps de malaltia $i$ es reincorporaven a la feina quan ja estaven curats del tot. Recordem que, per la duresa de l'ofici i les condicions de treball, es limitava a 40 anys l'edat d'ingrés. Tampoc no s'hi admetien persones físicament debils?

Malgrat tot, que sapiguem, hom desconeixia quines eren les tarifes dels salaris assignats als barquers de la platja de Barcelona i com s'aplicaven. Els preus que marca la llista regien per a tota mena d'embarcacions, grans i petites, i tenien la mateixa vigència tant a l'estiu com a l'hivern. Variaven, però, si les naus estaven fondejades fora de la tasca o àncora, és a dir, a mar obert, o dins d'aquella. També eren més elevades les taxes per operar en les barques cobertes que no en les descobertes; es considerava coberta sempre que ho fos per terç ab corredors de para scut a para scut ${ }^{10}$.

Calia utilitzar quinals ${ }^{11}$ e bragues $^{12}$ per descarregar saques de forment, ordis, civades $\mathrm{i}$ altres productes semblants $\mathrm{i}$ posar-les a les barques $\mathrm{i}$ transportar-les després a la platja per deixar-les en exut cinc passes, com ja hem dit.

La relació ens permet de conèixer quines devien ésser les mercaderies més corrents que eren objecte de comerç a la ciutat de Barcelona, perquè ja existia la previsió del cost dels treballs de càrrega i descàrrega. De determinats productes, ens n'indica la procedència: forment $\mathrm{i}$ civades de Tortosa $\mathrm{i}$ de tota la costa; llibants de Blanes; corda rodona i trunyella de València; taules i cabirons de Castello, i també files; cuiram castellà i de Portugal; roure de Flandes; draps fins francesos, anglesos i florentins; coto mapus ${ }^{13}$ de Malta; llana de Sardenya i de Barbaria; draps nostrats o de Lengadoch; cotó turquesch.

Ens interessa, especialment, subratllar l'embalatge amb el qual eren transportats els diversos productes (costals, sacs, gerres, botes e en altre manera) i les mesures respectives; fets que deduîm per la forma en què s'estableix la taxa a percebre per unitats transportables, que ben sovint

9 Ordinacions de 1480 dites dels barquers avellsw, vegeu nota 2.

10. Els para-escuts formen els dos costats estrets de l'escotilla i van col-locats en sentit de babord a estribord.

11 Conjunt de dos ternals combinats per alçar pesos grossos.

12 Peça de ferro que abraça o lliga altres dues peces.

13 Buata. Per al coneixement concret de les mercaderies objecte de comerç vegeu M. Gual Camarena, Vocabulario del comercio medieval, Barcelona 1976. 
equival a aquell fardell que pot portar un home (quiscun costal de coll de un home).

Així, el forment, els llegums, l'ordi i la civada es compten per salmes ${ }^{14}$, quantitat que equivalia aproximadament a tres quarteres i mitja de Barcelona; però també és transportat el forment en saques de mig cafís ${ }^{15}$ i en orri ${ }^{16}$.

$\mathrm{Vi}$, oli, graixa, sofre, tàrtar es transportaven en bótes, carratells ${ }^{17}$, terceroles $^{18} \mathrm{o}$ quarteroles ${ }^{19}$. El sucre podia arribar també en carratells o terceroles.

Gerres plenes de mel i d'oli. La formatgeria en orri, en sils o en cofi ${ }^{20}$. La sardina $\mathrm{i}$ anxova en barrils, els arenchs en bolas.

El cuiram es comptava per traques ${ }^{21}$, la pega per pans, el quitrà per ordres i el paper per bales.

Les alfabies de diverses mides (gran, mitjana, tercenca i petita), com també els cossis, servien per al transport de peces fabricades pels terrissers.

Diverses mercaderies s'embarcaven en sacs: avallana, gotzema ${ }^{22}$, pastell, recuyt, coto mapus; la duella i els riscles per somades ${ }^{2 \beta}$. Altres mercaderies eren taxades pel nombre: així fusts de ballestes e teles de ballestes, per quiscun centenar; l'arengade e rovellada, per miler; cordes, llibants i palomeres per unitats, encara que també es comptava per mola ${ }^{24}$.

Però una gran part era comptabilitzada en quintars, com és el cas de: espècies, drogues, laques, gomes, encensos, ceres, confits, brasils, indis i metalls, plom, estany, coure, 1laut6, ferro, acer, tatxeria, plates, fulles, fil de ferro i esmeril, obrats o no; també els merlussos i els congres. L'arròs i l'ametlló, per càrregues. La figa i la pansa eren comptades per guintar barbaresc.

Diverses mercaderies eren carregades a coll i es taxaven pel costal de coll de l'home, o bé pel cap de coll de l'home, bé d'una manuella o bé de dues.

Per bé que podríem, encara, adduir més citacions, creiem que el que hem dit és suficient per a una visió global del tema, i més tenint en compte que en donem a l'apèndix la transcripció íntegra.

${ }^{14}$ Per a les equivalències de mesures, CAPMANY, op. cit., II, p. 1.060-1.062, i el Diccionari català, valencia, balear d'ALCOVER MOLL.

${ }^{15}$ Mesura de capacitat, equivalent a vint-i-quatre barcelles.

16 Vegeu nota 20.

${ }_{17}$ Barril petit.

${ }^{18}$ Barril de cabuda mitjana.

19 Recipient per mesurar i tenir grans.

${ }^{20}$ De difícil identificació. El mot sil en català no es troba i en orri més aviat s'aplica a càrregues sense encaixar; creiem que no és el nostre cas, perquè afegeix o aitrament que no sia enbotade. El cofi, en canvi, és una mena de senalla planera d'espart.

${ }^{21}$ Feix o bala de cuirs.

22 Espigol

${ }^{23}$ Carrega d'un animal de bast que equival a tres quintars.

24. Munt de corda plegada en forma de voltes circulars superposades. 


\section{APÈNDIX}

Ara hoiats tot hom generalment per manament del honorable Balle ordonaren los consellers e promens de la dita ciutat per utilitat de la mercaderia e per bon stament de la dita ciutat que tots e sengles barquers o altres qui vullen barqueiar en la dita ciutat o en la plage de la mar de aquella haian e sian tenguts varar e entrar personalment, si requests ne seran per los honorables consols de la mar de la dita ciutat o per lur par, e fer entrar lurs missatges ab lurs barques ben armades en tots e sengles navili o navilis o fustes e carregar o descarregar aquelles mercaderias, robes o havers en o dels dits navilis o fustes sens contradictio alguna sots ban de.X. lliures Barchalonesas per quiscuna vegade que sera contrafet.

\section{Que lo llavador se puga retenir penyora}

rTEM, ordonaren los dits Cosellers (sic) e promens que tots e sengles mercaders o altres que faran carregar o descarregar mercaderias, robes o havers sian tenguts pegar de continent lo salari segons la tatxacio e ordinacio deius scrita als dits barquers $o$ a lur cullidor. E si nou faran que los dits barquers o lo lur cullidor se puxen retenir penyores per lur salari sens incorriment de alguna pena.

ITEM, ordonaren los dits consellers e promens que los dits barquers o lo dit cullir (sic) o altres qui rebut hauran los dits salaris sian tenguts e haian compartir aquell entre los qui treballat hi hauran e liurar a quiscu sa part de ço gue guanyat haura dins tres dies apres que sera dat compliment al carregar o descarregar de navili o fuste que carregat o descarregat hauran sots ban de.X. lliures barchinoneses per quiscuna vegade que sera contrafet.

ITEM, ordonaren los dits consellers e promens que sobre aço que deuen haver los dits barquers de las robes, mercaderias e havers qui.s descarregaran per ells o lurs misatges en la plage de la mar de Barchinona de qualsevol navilis grans o pochs e destiu com divern que stigan ormeiats fora la tascha o la anchora en obert sian servades tambe per los mercaders e altres qualsevulle persones com per los dits barquers les tatxacions seguents

\section{Co es per descarregar de naus}

Fforment e legums pagant per quiscuna salma general nou diners.

Ordi e civade comptant quatre salmes per tot a raho de nou diners. Vi, oli, graixa, soffra, tartar, totes coses qui vinguen en botes, carratells o terceroles (sic) o quartaroles, paguen a raho de bota dos sous. Dos sous. 
Tonells de fill de ballesta, per quiscu, tres diners. Tres diners. Sucres empero encara que vinguen en carratells o terceroles o en altre manera, paguen per quiscun quintar, tres diners.

Gerres de oli o altre qualsevol mercaderia engerrade paguen per quiscuna gerra valenciana plena e si sera maiors o menors paguaran o mes almenys a la dita raho de sis diners. Sis diners.

E que quantes de mel sian, comptats tres canters per duas gerras: fformatgeria en orri o en sils o en coffes o altrament que no sia enbotade, per quiscun quintar, dos diners e malla.

Species, drogues, laques, gomes, ensensos, corals, ceres, confits, brasils, indis, verdeh, cotonines, canamasseria ${ }^{25}$, lin, coto, turquesch, canem filat, canem scomes, canem fluix, stopes, comins, batafalues, totes lavors e semblants havers, per quiscun quintar, dos diners e malla.

Alums de qualsevol sort en orri o en saqueria, per quiscun quintar, tres diners.

Plom, stany, coure, leuto, ferre, acer, tatxaria, plates, fulles, fill de ferre e esmaril obrat e per obrar, per quintar, dos diners.

Roga, per quiscun quintar, dos diners.

Ffusts de ballestes e teles de balestes, per quiscun centenar, dotze diners.

Sal de qualsevol part, per quiscun centenar de corteres grosses, XXXV sous.

Testes mascles e fembres, per quiscun cap, sis diners.

Botes buydes, per quiscun cap, quatre diners.

Draps fins francesos, anglesos e florentins e semblants draps, per quiscun, dos diners.

Bales o fardells de barrets qui paguen a raho de nolit per bolum de draps, per quiscun drap al dit for, dos diners.

Draps nostrats o de lengadoch cruus o apparellats, per quiscun, un diner. Un diner.

Roure de Flandes per quiscun born, un diner. Un diner.

Arenchs per quiscuna bala, tres diners. Tres diners.

Congres per quiscun quintar, dos diners e mala. Dos diners e Malla.

Merlussos, per quiscun quintar, dos diners c malla. Dos diners e malla.

Arangade e rovellade, per quiscun miller, tres diners. Tres diners.

Cassia fistola, per quiscun quintar, quatre diners. Quatre diners.

Coto de Síria e de Alexandria, per quiscun quintar, quatre diners.

Coto mapus de Malta, per quiscun sach, sis diners.

Coto faldeta o filat a raho entes dos sachs per .1. sach de coto mapus, sis diners.

Pastells, bordats, datils, paper, canamaceria, teles, fustanis, caixes de miralls, pelliceria embalade, cuyram, lana de Cerdenya, lana de Barberia, avallana, nous, castanyes, amenles, vedrieres, seu e per semblants havers per quiscun cap de coll de 1. home, quatre diners.

E per quiscun coll de una manuella dels dits havers, sis diners.

ITEM per quiscun cap de coll de. II. manuelles dels dits havers, vuyt diners.

Cuyram castella, per quiscuna traca de .X. cuyrs bovins, deu diners.

25 Abreviatura de dificil interpretacio. 
Cuyrs de vadells per quiscuna traca, .v. diners. Sinch diners.

Cuyram de Lisbona e de Purtogal per quiscuna traca de .X. cuyrs bovins, vIII. diners. Vuyt diners.

Cuyrs de vadells de aquella sort per quiscuna traca, quatre diners.

Pega per quiscun pa, dos diners.

Alquitra per quiscun odre, dos diners.

ITEM ordonaren los dits consellers e promens que sobre aço que deuen haver los dits barquers de las robes, mercaderies e havers qui-s carregaran per ella o lurs missatges en la plage de la mar de Barchinona en qualsevol navilis, grans o pochs, e axi d'estiu com d'ivern, que stigan ormegats fora la tascha o a la anchora en uber, sian servades axi per los mercades e altres personas com per los dits barquers les tatxacions seguents:

Drapades paguen per quiscun drap, un diner. 1 diner.

Corals, ferre, amenlo, gotzema, laques, merceria, erros, alums, vermello, saffra, gomes, ceres, argent viu, soffre, tartar, species, cotons turqueschos e semblants havers de [pesue?] per quiscun quintar, quintar (repetit), dos diners.

Coffol per quiscuna coffa, II. diners. Dos diners.

Fferre, tatxeria, plom, coure, stany, acer, lauto, indi, arnes e tot altre matall obrat e per obrar per quintar.II. diners e malla.

Gerres plenes de olis ab la stiba per quiscun centenar a raho de trentatres sous.

Gerres plenes de mels ab la stiba per quiscun centenar a raho de vint sous.

Canters de mel, son comtats tres per dos gerres.

Avallana per quiscun sach, sis diners.

Gotzema per quiscun sach, sis diners.

Alfabies grans plenes de obra de terra per cascuna, setze diners.

Alfabia migana, setze diners.

Alfabia terrenca, nou diners.

Cossis de March ab obra de terra, sis diners.

Cossi gran ple de obra de terra, nou diners.

Alfabies petites ab obra de terra per quiscuna quatre diners.

Cuyram de remudes, boldrons, cuyram gros o menut, lanes, sayes, teles, bordats, canamasseria, fustanis, cotons, pelliceria, crua ${ }^{26}$ o adobada. E semblants havers per quiscun costal de coll de .1 . home, quatre diners.

E per quiscun costal de coll de una manuella, sis diners.

E per quiscun costal de coll de dues manuelles, vuyt diners.

Vin per quiscuna bota, dos sous.

Rests de XL. libants a ops d'ormeyar o per carregar per quiscun, quatre sous. Quatre sous.

E de palomera de aquella sort, sis diners.

Rests de trenta libants per quiscun, sis diners.

E de quiscuna palomera de aquella sort, quatre diners.

Rests tortissos de la manor sort per quiscu, devuit diners.

26 Diu ceua. 
E de quiscuna palomera de aquella sort, quatre diners. Quatre diners.

Palomeres de quarante e de sinquante e de sexanta e libants e altres coses d'espart que paguen per dotzena a nolit fahent compte dels libants de vuyt canes e totes altres coses d'espart e de palma paguen axi com del descarregar de las barques ço es per dotzena, tres diners.

ITEM ordonaren los dits consellers e prohomens que sobre so que deuen haver los dits barquers de las robes, mercaderias o havers qui-s descarregan per ells o per lurs missatges en la plage de la mar de Barchinona axi d'estiu com d'ivern de qualsevol fustes ormeyades dins la tascha e la barba de la tasca sian servades axi per los mercaders e altres persones com per los dits barquers les tatxacions seguents.

\section{Per descarregar de barques}

De forment de Tortosa e de tota la costa per quiscuna saca de mig caffis, de fuste descoberta, quatre diners.

E de fusta coberta, per quiscuna saca de mig caffis, sinch diners.

Sivades de Tortosa compten sinch per quatre a raho de quatre diners, en fuste descoberta. Quatre diners.

E de fusta coberta a la dita raho, sinch diners.

Forment en orri de Tortosa, en barca descoberta, per quiscun caffis, dotze diners.

De barca coberta, per quiscun caffis, quatorze diners.

Sivade al dit for reduhint sinch mesures per quatre, quatorze diners.

Fforment en orri de quiscuna altre part per quiscuna quortera, dos diners.

Sivade en orri de qualsevol altre part per quiscun centenar, quatorze diners.

Vin per quiscuna bota exaugade de la scuma de la mar en la sorra, quatorze diners.

E si pugeran la bota exaugade de la scuma es part de la plaça on lo mercader volra, per quiscuna bota, devuyt diners.

Miges botes e carratells axi mateix segons mes o menys al dit for.

Ferre per quiscun quintar tres malles, I. diner malla.

Acer, per quiscun balo, dos diners. Dos diners.

Plom, stany, coure, leuto, tatxeria, plates, fulla, fil de ferre, esmarill e tot semblant matall obrat e no obrat, per quiscun quintar tres malles, 1, diner malla.

Sardina, anxova per quiscun barril, un diner.

Duella, riscles per quiscuna somade, sis diners.

Paper, per quiscuna bala, tres diners.

Canamasseria, teles, fustanis, bordats, caixes de pintes, caixes de miralls, pelliceria ambalade, cuyram, lana de Serdenya, lana de Barbaria, datils, avellana, nous, castanyes, amelles, vevedrieres, seu. E semblants havers per quiscun cap de coll de 1. hom, cinch diners.

De una manuella, cinch diners.

De dues manuelles, set diners.

Pastell, per quiscun sach, tres diners. 
Recuyt, per quiscun sach, dos diners.

Avallana, per quiscun sach, quatre diners.

Ffiga e pansa, per quiscun quintar barbaresch, dos diners.

Batafalua, comi e totes lavors de quiscun costal comu, quatre diners.

Cuyram de boquines de cent pells, sis diners.

Lata de spart, per dotze dotzenas, dotze diners.

Libants de exanega per mola tres malles, 1 . diner malla.

Libants de Blanes, per mola, 1 diner.

Libants de nança, tres moles, dos diners.

Erros per quiscuna carrega, quatre diners.

Costal de sarrias, sis diners.

Coffres, per quiscun, sis diners.

Alfabia gran de obra de terra. per quiscuna, setze diners.

Alfabia migana per quiscuna, dotze diners.

Alfabia tercenca ${ }^{27}$, per quiscuna, nou diners.

Alfabies petites, per quiscuna, quatre diners.

Cossis de obres de terra per.1. March, sis diners.

Cossi gran plen de obra de terra, nou diners.

Amello, per quiscuna carrega, quatre diners.

Pega e alquitra per quiscun pa o odre, dos diners.

Taules de Castello de .X. en .XII. palms, per quiscuna dotzena, quatre diners.

Cabirons de Castello sotils, per quiscuna dotzena, einch diners.

Cabírons de Castello dels maiors, per dotzena, sis diners.

Ffiles de Castello, per dotzena, dotze diners.

Garroffa per quiscun quintar a nolit posant-la al loch acostumat, dos diners.

Sal per quiscun centenar de corteras a la mesura grossa, vint e set sous.

Rests de .XI. libants, per quiscu, tres sous.

Rests de .XXX. libants per quiscu, devuyt diners.

Rests de menor sort, dotze diners.

Palomeres de quoerna per una dotzena, tres diners.

Palomeres de terna tres peces per dues dotzenas, set diners.

Palomeres de LX peces de VIII. canes van .II. per una dotzena, tres diners e malla.

Palomeres de $\mathrm{L}$ passes van tres per una dotzena, tres diners e malla.

Palomera de $X X X X$ passes ptimers van $V$. per una dotzena, tres diners e malla.

Libants de sinquena van VI. per una dotzena, tres diners e malla.

Libants de quoerna van .VII, per una dotzena, tres diners e malla.

Libants de terna van. . per una dotzena, tres diners e malla.

Libants de .VIII canes van .XII. per .1. dotzena, tres diners e malla.

Trunyella prima. IIII. centenars per .1. dotzena, tres diners e malla.

Dos centenars de trunyella de rigot, van per una dotzena, tres diners e malla.

Corda rodona de Valencia lo centenar, quatre diners.

Corda rodona migana van .II. centenars per .1. centenar, quatre diners.

${ }^{27}$ Es nota una certa vacil lació en l'escriptura, i interpretem tercenca refererit a la capacitat. 
Trunyella de Valencia, 10 centenar, tres diners.

Faix de manuella de sarrias buydes, vuyt diners.

Stores de payol, la dotzena, quatre diners.

Com segons ordinacions per los honorables consellers e prohomens de la ciutat de Barchinona qui lavors eran en temps passat fetes e ab veu de crida per los lochs acostumats de la dita ciutat publicades de manament del honorable vaguer de la dita ciutat los barquers de la dita ciutat haguessen e rebessen $e$ haian e reben per descarregar certes robes e havers certes quantitats de diners, ço es de barquers ${ }^{28}$ cubertes maior quantitat e de barques descobertes menor quantitat segons les tatxacions en les dites ordinacions contengudes. E per ço com en lo present temps hic ha barques en les quals son fets axi a popa com a proha talems ab certs corredors assats grans per los quals se causa dubte si la tal barcha si sera dita cuberta 0 descuberta. E per vigor de la retencio acostumade posar en ordinacions fetes e qui-s fan per consellers e prohomens la declaracio de semblant dubte se pertangue als honorables consellers e prohomens de la dita ciutat per ço dijous a VI. del mes de juny del any M.CCCC XXXVII los honorables mossen Joan Buçot, mossen Guillem de Vallseca, mossen Pere Arnau Pol e mossen Jacme Ros consellers 1. any present de la dita ciutat ab consell de certs prohomens de cascuns staments e specialment de prohomens experts en semblants e altres actes maritims constituits dins lo ort de la casa del consell de la dita ciutat declarans lo dupte desus dit ordonaren tant com es solament al interes dels barquers que de tota barca la qual sia cuberta per terç ab corredors de para scut a para scut sia hauda e reputade per barca coberta e no per barca descoberta axi que n sia demanat e haut lo dret segons les tatxacions dessus fetes de barques cobertes.

ITEM ordonaren los dits consellers e prohomens de la dita ciutat avistant a les dites coses ordonades en lo passat que d aci anant tots e sengles barquers qui descarregaran saques de forment, ordis civades e altres qualsevol fays hagen sian tenguts descarregar ab quinal e ab bragues e metre-ho en lurs barques sots ban de V. sous. a quiscu e per quiscuna vegade que sera fet lo contrari.

ITEM ordonaren los dits consellers e prohomens de la dita ciutat quals dits barquers com trauran de lurs barques descarregant qualsevol robes e mercaderias de qualsevol ley o specia sian, haian e sian tenguts posar aquelles cinch passes en exut luny de la mar sots ban de .v, sous. a quiscu e per quiscuna vegade que sera fet lo contrari.

ITEM ordonaren los dits consellers e prohomens de la dita ciutat que-ls dits barquers com trauran de lurs barques descarregant qualsevol robes e mercaderias de qualsevol ley o specia sian, haian e sian tenguts posar aquelles cinch passes en exut luny de fa mar sots ban de . $v$, sous. a quíscu e per quiscuna vegade que sera fet lo contrari.

Dels quals bans sian fetes tres eguals parts, la una de las quals sia del official qui.n fara la execucio e la altra part del acusador. E la restant terça part de la obra dels murs e dels valls de la dita ciutat Retenense, etc.

28 Per barques. 